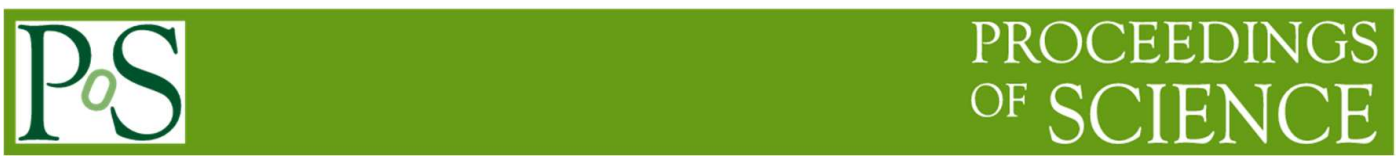

\title{
Wide Angle Spherical Neutron Polarimetry at Oak Ridge National Laboratory
}

\author{
Silva, Nicolas ${ }^{1}$ \\ Neutron Science Directorate, Oak Ridge National Laboratory \\ Oak Ridge, TN 37831 United States \\ E-mail: silvaneornl.gov
}

\section{Wang, Tianhao}

Institute of High Energy Physics, Chinese Academy of Sciences Beijing 100048, China

Dongguan Neutron Science Center, Dongguan 523803

\section{Jiang, Chenyang}

Neutron Science Directorate, Oak Ridge National Laboratory Oak Ridge, TN 37831 United States

E-mail: jiangceornl.gov

\section{Li, Fankang}

Neutron Science Directorate, Oak Ridge National Laboratory Oak Ridge, TN 37831 United States

E-mail: frankli@ornl.gov

\section{Debeer-Schmitt, Lisa}

Neutron Science Directorate, Oak Ridge National Laboratory Oak Ridge, TN 37831 United States

E-mail: debeerschmlmeornl.gov

\section{Matsuda, Masaaki}

Neutron Science Directorate, Oak Ridge National Laboratory Oak Ridge, TN 37831 United States

E-mail: matsudameorn1.gov

\section{Ruff, Jillian}

Neutron Science Directorate, Oak Ridge National Laboratory Oak Ridge, TN 37831 United States

E-mail: ruffjmeornl.gov

\section{Pynn, Roger}

Department of Physics, Indiana University

Bloomington, IN 47405 United States

E-mail: pynnreornl.gov

\section{Tong, Tony}

Institute of High Energy Physics, Chinese Academy of Sciences

\section{${ }^{1}$ Speaker}

(C) Copyright owned by the author(s) under the terms of the Creative Commons 
Beijing 100048, China

Dongguan Neutron Science Center, Dongguan 523803

\section{Winn, Barry}

Neutron Science Directorate, Oak Ridge National Laboratory

Oak Ridge, TN 37831 United States

E-mail: winnbleornl.gov

Spherical Neutron Polarimetry (SNP) analyzes complex magnetic structures through distinguishing contributions from nuclear-magnetic interference and chiral structure in addition to nuclear magnetic scattering separation. This analysis is achieved through determining all components in the polarization transfer process. Currently, wide-angle SNP is being realized at Oak Ridge National Laboratory (ORNL) for multiple beamlines including: the polarized tripleaxis spectrometer (HB-1) and general-purpose small angle neutron scattering instrument (CG-2) at the High Flux Isotope Reactor (HFIR), as well as the hybrid spectrometer (HYSPEC) at the Spallation Neutron Source (SNS). The SNP device consists of three units: incoming/outgoing neutron polarization, sample environment and a zero-field chamber. The incoming/outgoing neutron polarization regions use high-T_c superconducting YBCO films and mu-metal to achieve full control of neutron polarization. The sample environment is an evaporation refrigerator with a customized tail piece placed into the zero-field chamber. The device has been fully assembled and tested at the University of Missouri Research Reactor (MURR).

The 18th International Workshop on Polarized Sources, Targets, and Polarimetry, PSTP2019 23 -27 September, 2019

Knoxville, Tennessee 


\section{Introduction}

Polarized neutron scattering is a powerful tool in studying magnetism in materials because of the strong interaction between neutron and electron spins. Various polarized neutron techniques such as longitudinal and XYZ polarization analysis have been widely used in major neutron facilities around the world.

The Blume-Maleev equations [1,2] are the master equations describing polarized neutron scattering as shown in Eq. 1 and 2:

$$
\begin{gathered}
I=N N^{*}+\boldsymbol{M}_{\perp} \cdot \boldsymbol{M}_{\perp}^{*}+i \boldsymbol{P}_{i} \cdot\left(\boldsymbol{M}_{\perp}^{*} \times \boldsymbol{M}_{\perp}\right)+\boldsymbol{P}_{i} \cdot\left(N \boldsymbol{M}_{\perp}^{*}+N^{*} \boldsymbol{M}_{\perp}\right) \\
\begin{aligned}
\boldsymbol{P}_{f} I= & \boldsymbol{P}_{i} N N^{*} \\
+ & \boldsymbol{M}_{\perp}\left(\boldsymbol{P}_{i} \cdot \boldsymbol{M}_{\perp}^{*}\right)+\boldsymbol{M}_{\perp}^{*}\left(\boldsymbol{P}_{i} \cdot \boldsymbol{M}_{\perp}\right)-\boldsymbol{P}_{i}\left(\boldsymbol{M}_{\perp} \cdot \boldsymbol{M}_{\perp}^{*}\right) \\
& \quad i\left(\boldsymbol{M}_{\perp}^{*} \times \boldsymbol{M}_{\perp}\right) \\
& +N^{*} \boldsymbol{M}_{\perp}+N \boldsymbol{M}_{\perp}^{*}+i \boldsymbol{P}_{i} \times\left(N \boldsymbol{M}_{\perp}^{*}-N^{*} \boldsymbol{M}_{\perp}\right)
\end{aligned}
\end{gathered}
$$

In these two equations, $I$ is the scattering intensity proportional to the scattering cross section, $\boldsymbol{P}_{i}$ the incident neutron polarization, $\boldsymbol{P}_{f}$ the final neutron polarization, $N$ the nuclear structure vector, and $\boldsymbol{M}_{\perp}$ the magnetic interaction vector which is the projection of the magnetic structure factor onto the plane of the scattering vector. The first term in Eq. 1 describes the nuclear scattering, the second term the traditional magnetic scattering, the third term the chiral magnetic interaction and the last one the nuclear-magnetic interference. Eq. 2 describes the change of neutron polarization under the four interactions in Eq. 1 and shows that in general the neutron polarization will change both in magnitude and direction upon scattering from a magnetic material. By defining 3 polarization axes as $\mathrm{x}$ parallel to the scattering vector $\mathrm{Q}$, $\mathrm{z}$ perpendicular to the scattering plane (usually the vertical direction) and y completing the right-handed coordinate system, and then measuring the scattered neutron polarization with the incident polarization along each of the polarization axes, Eq. 2 can be summarized with a polarization matrix which is what is actually measured in a SNP experiment:

$$
P_{f, i}=\left[\begin{array}{ccc}
\frac{N^{2}-M^{2}-2 \operatorname{Im}\left(M_{\perp y} M_{\perp z}^{*}\right)}{I_{x}} & \frac{-2 \operatorname{Im}\left(N M_{\perp z}^{*}\right)-2 \operatorname{Im}\left(M_{\perp y} M_{\perp z}^{*}\right)}{I_{y}} & \frac{2 \operatorname{Im}\left(N M_{\perp y}^{*}\right)-2 \operatorname{Im}\left(M_{\perp y} M_{\perp z}^{*}\right)}{I_{z}} \\
\frac{2 \operatorname{Im}\left(N M_{\perp z}^{*}\right)+2 \operatorname{Re}\left(N M_{\perp y}^{*}\right)}{I_{x}} & \frac{N^{2}-M^{2}+2 \operatorname{Re}\left(M_{\perp y} M_{\perp y}^{*}\right)+2 \operatorname{Re}\left(N M_{\perp y}^{*}\right)}{I_{y}} & \frac{2 \operatorname{Re}\left(M_{\perp y} M_{\perp z}^{*}\right)+2 \operatorname{Re}\left(N M_{\perp y}^{*}\right)}{I_{z}} \\
\frac{-2 \operatorname{Im}\left(N M_{\perp y}^{*}\right)+2 \operatorname{Re}\left(N M_{\perp z}^{*}\right)}{I_{x}} & \frac{2 \operatorname{Re}\left(M_{\perp y} M_{\perp z}^{*}\right)+2 \operatorname{Re}\left(N M_{\perp z}^{*}\right)}{I_{y}} & \frac{N^{2}-M^{2}+2 \operatorname{Re}\left(M_{\perp z} M_{\perp z}^{*}\right)+2 \operatorname{Re}\left(N M_{\perp z}^{*}\right)}{I_{z}}
\end{array}\right] .
$$

The diagonal terms of this polarization matrix give information of the traditional nuclear scattering and magnetic scattering while the off-diagonal terms contain chiral contributions and nuclear-magnetic interreference. The longitudinal and XYZ methods can measure the diagonal terms in the polarization matrix but leave out the off-diagonal terms. Only SNP provides a way to accessing the off-diagonal components and thus allows researchers to extract the complete information in the scattering. Figure 1 shows a schematic between a traditional technique vs. SNP. 
(a)

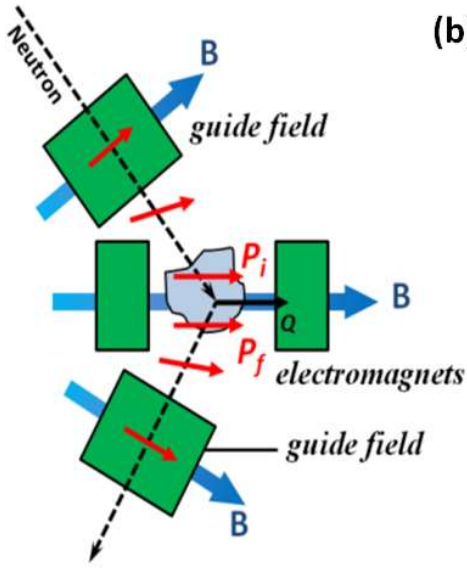

(b)

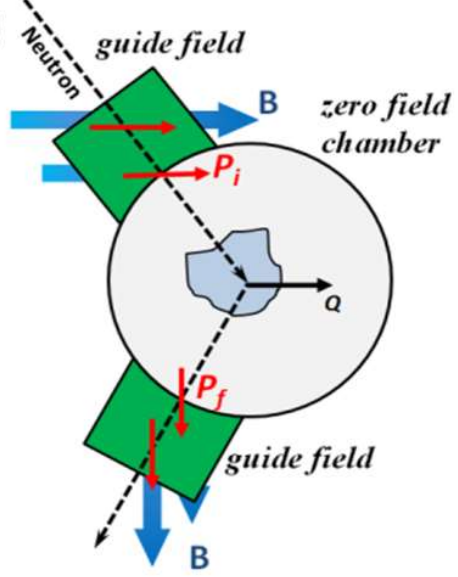

Figure 1 - a) A schematic representation of traditional polarization analysis, note the continuous field throughout the neutron direction. b) A schematic representation of spherical neutron polarimetry, the introduction of the zero-field chamber allows for the decoupling of incoming and outgoing neutron polarization.

In traditional techniques there is a guide field present throughout the entire neutron path to help maintain neutron polarization. Therefore, the traditional neutron polarization analysis techniques only measure the final polarization parallel/antiparallel to the incoming polarization as shown in Figure 1(a), and the measured polarization is only the projection of the actual polarization to the direction defined by the guide field. They cannot differentiate between the true depolarization and the rotation of the neutron polarization in the scattering. In comparison, SNP decouples the incoming and outgoing neutron polarizations by setting up a zero-field region at the sample position (Figure 1(b)), which enables measurement of all 9 components in the polarization matrix.

It is vital for SNP to have precise neutron polarization control for both incoming and outgoing neutrons which directly determines the accuracy of the results. The polarization manipulation is usually achieved through a combination of adiabatic and strict non-adiabatic neutron polarization transitions (Larmor precession). Highly accurate neutron polarization control relies on a sharp magnetic field boundary between each transition section, i.e., the boundary between the adiabatic transition section and the non-adiabatic transition section as well as the boundary between the non-adiabatic transition section and the zero-field chamber. The best solution to set up such a boundary is to use superconducting films by taking advantage of the Meissner effect. In Cryopad, an SNP device developed at Institut Laue-Langevin (ILL), niobium superconducting films (Tc $<$ $10 \mathrm{~K}$ ) are used which are cooled with liquid helium. As a comparison, we have developed an SNP device at Oak Ridge National Laborotary (ORNL) based on high-Tc superconducting YBCO films (Tc $\sim 93 \mathrm{~K}$ ). The application of the high-Tc films simplifies the overall design and makes the device more compact to maximize its portability and compatibility across different neutron instruments.

\subsection{Design of the SNP device}

The wide-angle design for our device is based on an existing small angle SNP development (CryoCUP) [5]. It will be used on the Polarized Triple-Axis Spectrometer (PTAX) and the General-Purpose Small Angle Neutron Scattering instrument at the High Flux Isotope Reactor (HFIR). Lastly, the device will be expanded for use on the Hybrid Spectrometer (HYSPEC) at the Spallation Neutron Source (SNS).

Our device, shown schematically in Figure 2, can be divided into three sections: the incoming/outgoing neutron polarization control, the zero-field chamber, and the sample environment. 


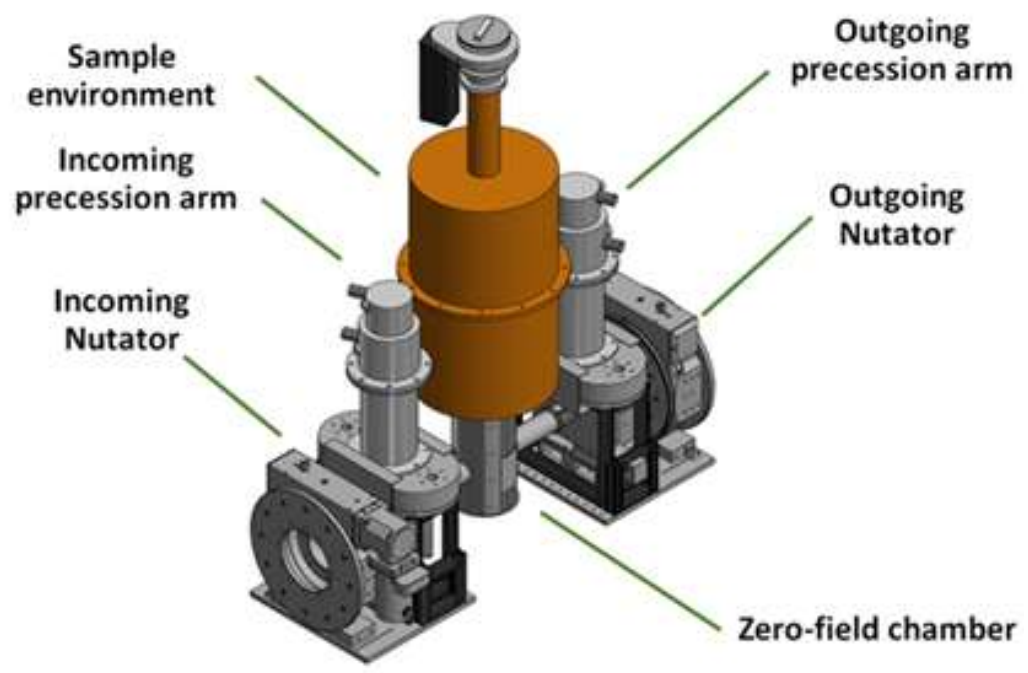

Figure 2 - Overall 3-D model of the entire device

The sample environment is a custom evaporation refrigerator more commonly known as an orange cryostat. It will come equipped with a non-magnetic goniometer, AttoCube $\odot$ ANGt10, to allow for precise alignment of the sample. The zero-field chamber (magnetic field inside $<10$ $\mathrm{mG})$, shown in Figure 3, is made entirely out of mu-metal. The chamber is made of concentric cylinders constructed in a manner that allow for the outgoing 'tunnel' to rotate a total of 85 degrees. The incoming zero field chamber arm is set to remain in a fixed position, while the downstream arm is free to rotate from -5 to 80 degrees for the wide-angle coverage.

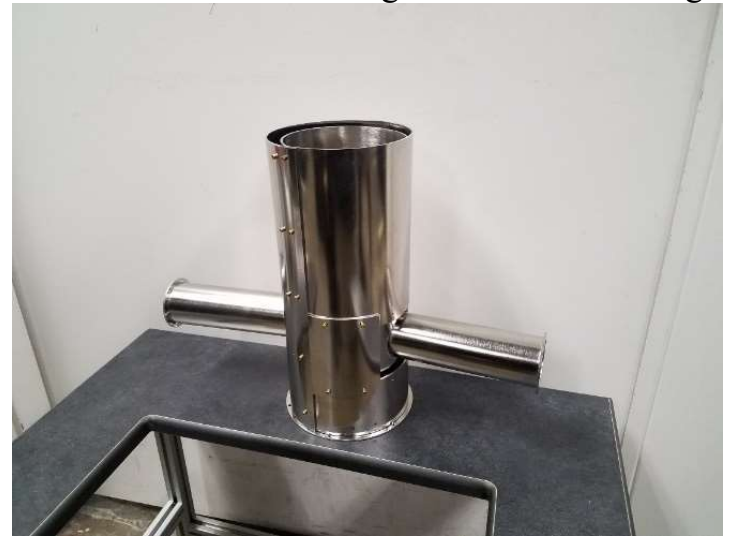

Figure 3 - The Assembled Zero-Field Chamber

The neutron polarization control in this design adopts a two-step approach on both the incoming and outgoing sides, except the order is reversed. For the incoming neutron polarization, demonstrated in Figure 4(a), it is first guided by a solenoid field $\mathbf{B}_{1}$ along the $\mathrm{x}^{\text {' direction and }}$ then adiabatically transferred into the y'-z' plane defined by a Meissner shield. The Meissner effect forces the magnetic field $\mathbf{B}_{2}$ to be parallel to its surface while the direction within the plane, defined as the polar angle $\theta$ in the Bloch sphere in Figure 4(b), is controlled by the nutator magnetic field, which can be rotated around the x' axis. The Meissner shield also creates a sharp magnetic field shift on each side, which allows a vertical field $\mathbf{B}_{3}$ to be applied between two Meissner shields. The vertical field $\mathbf{B}_{3}$ is generated by a pair of coils between the two Meissner shields, shown in figure $5 \mathrm{~b}$, so that its magnitude can be controlled by the current applied through. The combination of the controlled magnetic field $\mathbf{B}_{3}$ and the fixed distance between the two 
Meissner shields creates a controlled neutron polarization precession that defines the azimuthal angle $\phi$. Thus, in the coordinate system defined by x'-y'-z', through first control of the angle $\theta$ and then $\phi$ the incoming neutron polarization is well defined before entering the zero-field sample chamber. For outgoing neutrons, the order of the neutron precession and adiabatic transition is reversed.
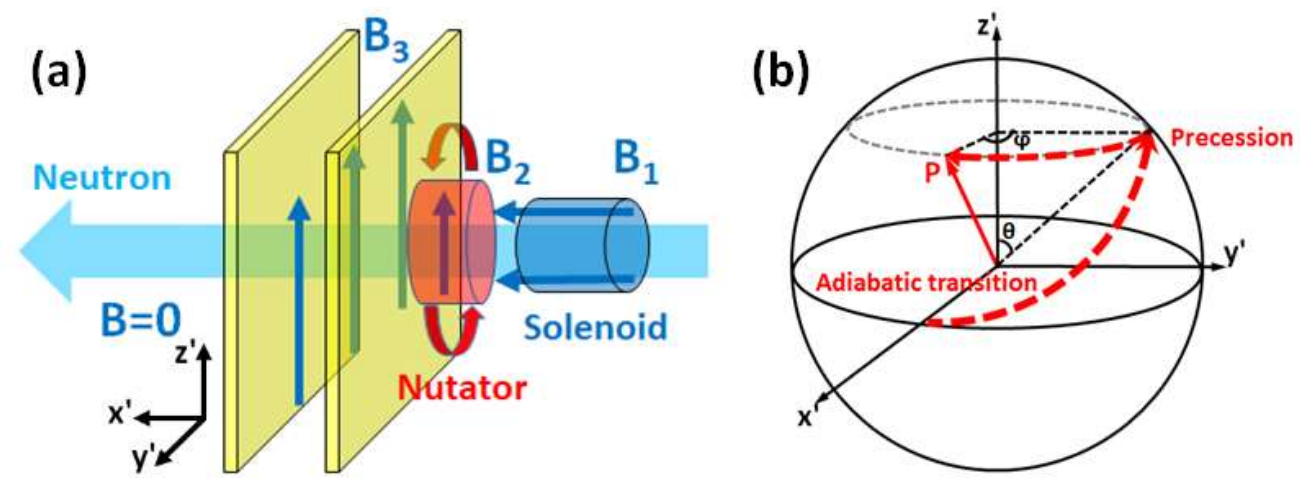

Figure 4 - a) Schematic representation of neutron polarization control where B signifies magnetic fields. b) Incoming neutron polarization movement on a Bloch sphere when a neutron travels through the magnetic field setup in (a).

To achieve this polarization control two devices were developed: the nutator, and the precession region. The nutator is essentially a rotatable magnetic guide field. It is a pair of iron pole pieces with copper wrapped around the base attached to a cylinder that is nested within a Huber goniometer as seen in Figure 5 below. This allows for the field generated from the pole pieces to be oriented in either the vertical or horizontal position (similarly to the field B2 in Figure 4(a)). The nutator also contains a solenoid to act as a guide field and maintain neutron polarization.

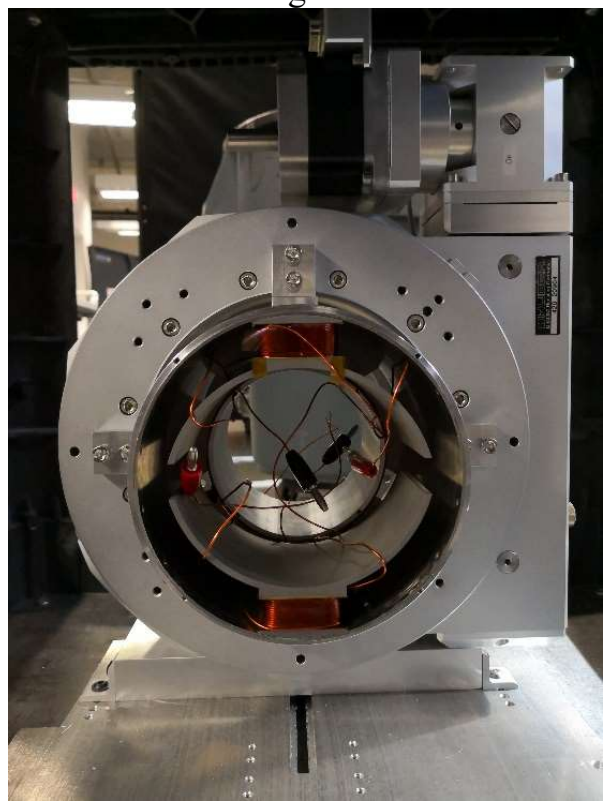

Figure 5 - Front view of the nutator, note the pole pieces in the vertical field position but can be rotated into any angle from the Huber goniometer

The precession region is represented as the two superconducting films, made from YBCO, with a field that can be generated between them. The actual parts are shown attached to the single stage closed circuit refrigerator (CCR) in Figure 6. The field is generated by a pair of coils wound with 
superconducting tape, also made from YBCO, that can have current applied to generate a vertical field to cause precession of the neutrons as they pass through.

A novel feature of our design is using YBCO as the superconducting material to act as the Meissner shield. The YBCO is a thin film $(200 \mathrm{~nm})$ deposited on a single crystal sapphire $(9 \mathrm{~cm}$ $\mathrm{x} 10 \mathrm{~cm}$ ). The performance of this film has already demonstrated to produce a reliable Meissner effect at temperatures below $93 \mathrm{~K}$ without introducing a noticeable transmission loss $[6,7,8]$. Additionally, the YBCO has simplified the cooling requirements for the device since it has a high superconducting temperature of $93 \mathrm{~K}$. This is easily accommodated by a single stage CCR which can take the temperature down to as low as $24 \mathrm{k}$.
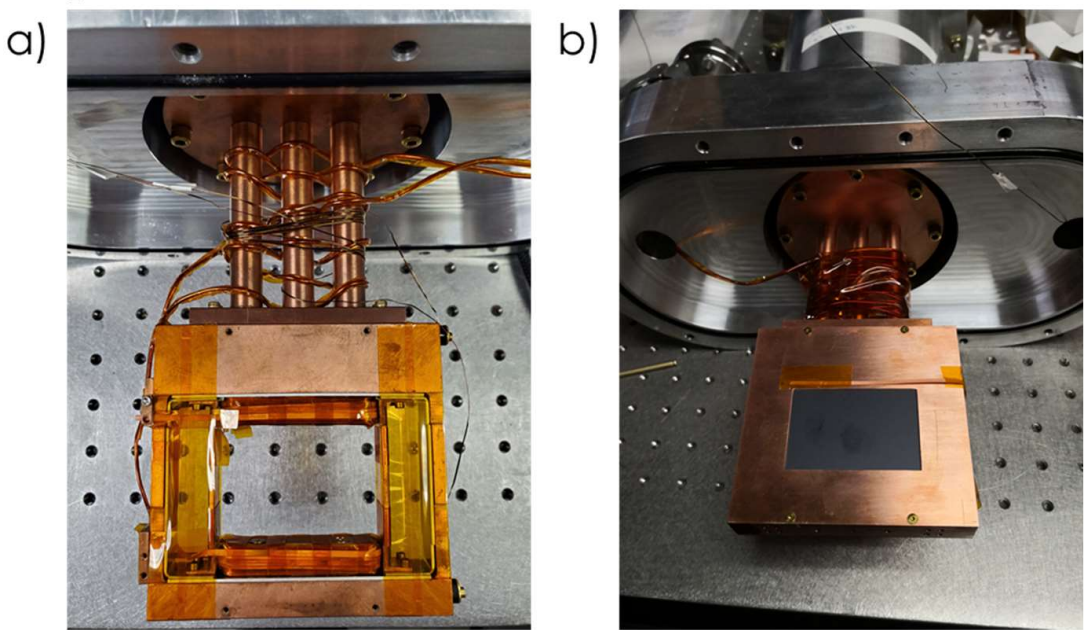

Figure 6-a) Superconducting coils attached to the precession assembly without the YBCO films b) Fully assembled precession region with the YBCO films shown through the square window

\subsection{Tests and Results}

The device was fit and set to run on beamline HB-1 at the high flux isotope reactor (HFIR) as seen in Figure 7, however; due to the shutdown of HFIR the device was unable to be tested there at this time.

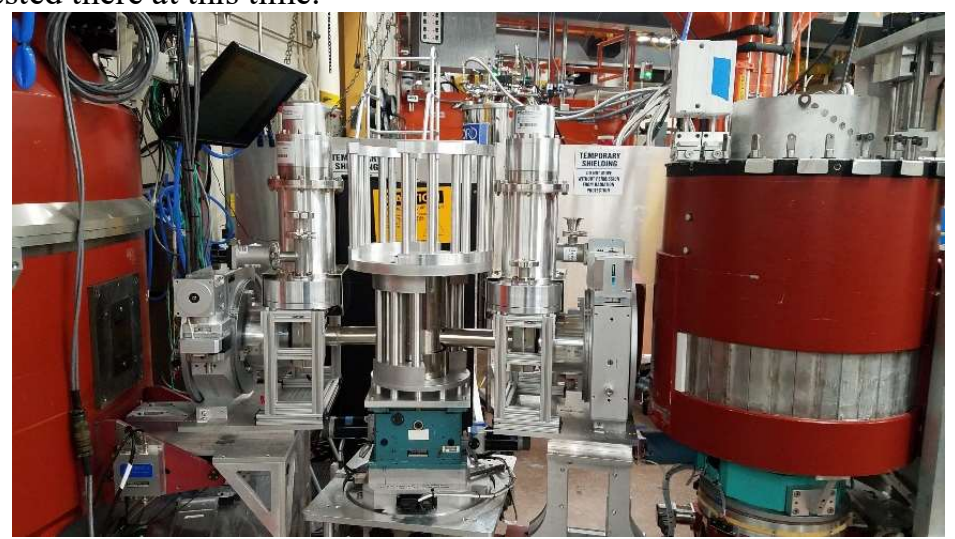

Figure 7 - Test fit and device setup on HB-1, the only missing component is the orange cryostat

The initial online test of the device was on the Double-Axis Powder Diffractometer $2 \mathrm{X}-\mathrm{C}$ at the University of Missouri Research Reactor (MURR). The beamline was reconfigured to fit the SNP device. Figure 8 shows the device setup on the beamline. Essentially, a table made from 80/20 was constructed so that an x-rail system could be attached and then our device components 
mounted onto the rail.

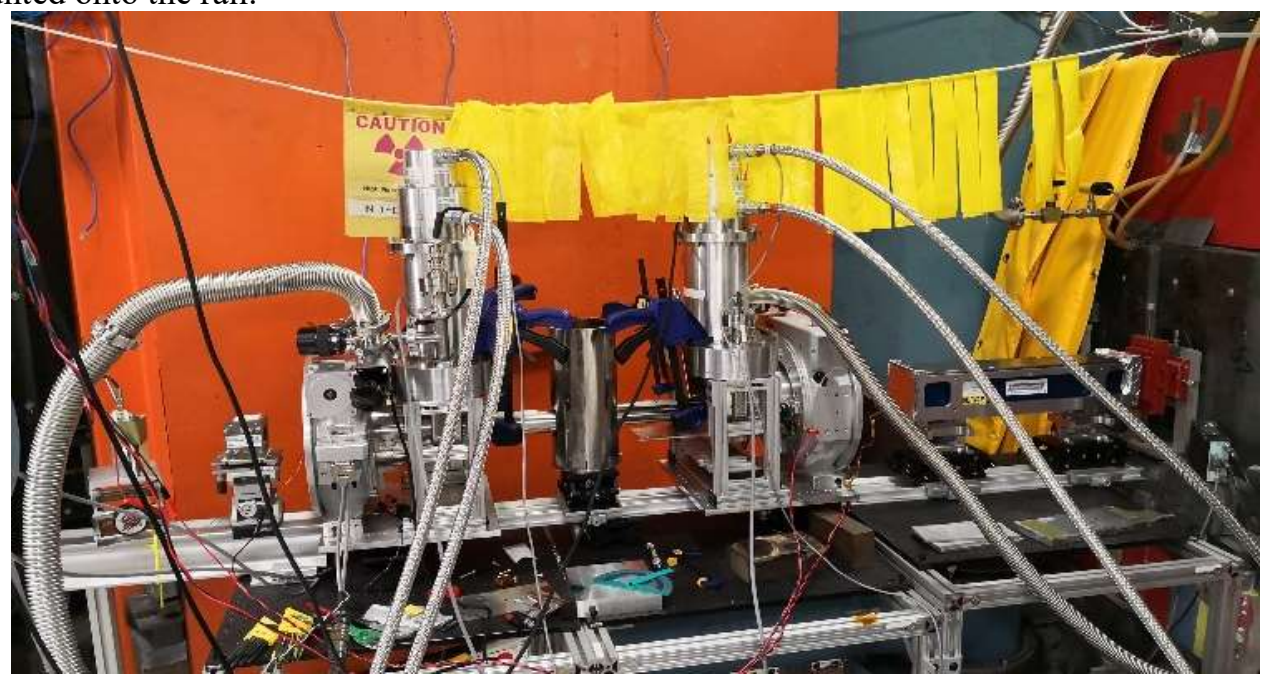

Figure 8 - The SNP device setup, the only missing component is also the orange cryostat

The neutron wavelength coming from the beam port is 4.3 Angstroms. On the incoming side of the neutrons was a V-cavity supermirror polarizer followed by the device and on the outgoing side an S-bender supermirror was used as the analyzer. Due to the limitations in infrastructure at MURR the orange cryostat could not be used, and the downstream arm was fixed at the straight position. Since the orange cryostat was unable to be used regular samples could not be tested. So a test solenoid was created and placed into the zero field chamber at an angle to act as a sample, demonstrated in Figure 9.

$\mathrm{a}$

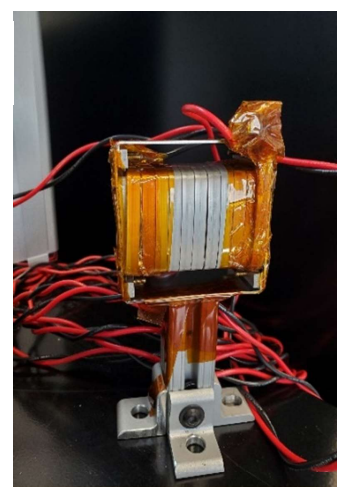

b

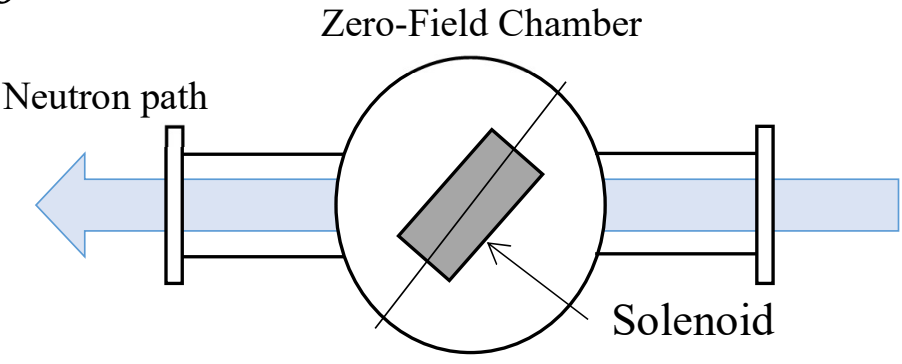

Figure 9 - a) Test solenoid created to act as a sample for experiments at MURR, it was wound with aluminum wire and encased with some Mu-metal. b) Top-view showing the placement of the solenoid within the zero field chamber, note the angle displacement

The first measurements made were transmission measurements with nothing placed in the zerofield chamber. Alignment between the upstream and downstream nutators as well as the upstream and downstream precession regions was performed. A precession scan was taken for both precession regions was also done. Figure $\mathbf{1 0}$ shows one of the precession scans. It is important to note that the minimum occurs at 0 current and the period is symmetrical. This indicates that there was very little additional polarization rotation in the zero-field chamber. The components for a polarization matrix were measured and shown in Table 1.

The next set of measurements were performed by placing a solenoid in the zero-field chamber to serve as a sample. The solenoid was placed inside the zero-field chamber at an angle (30 degrees relative to the neutron direction), as shown in Figure 9(b) and the polarization matrix components were measured, these results are also shown in Table 1. 


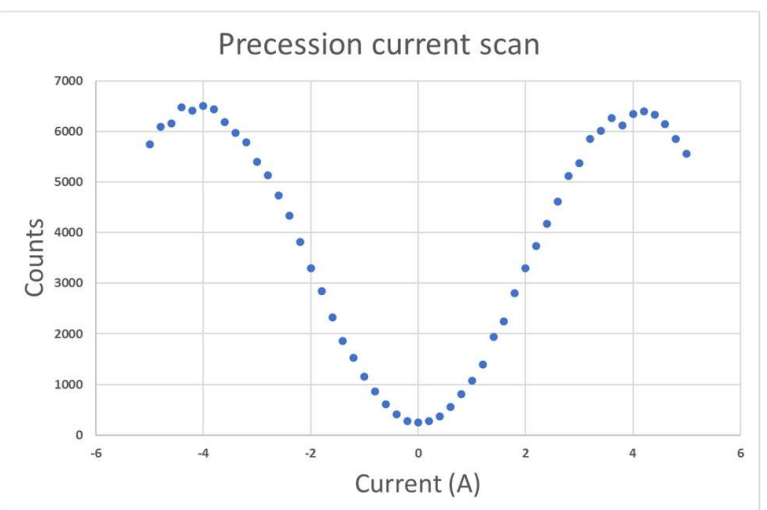

Figure 10 - Precession scan results from -6 Amps to +6 Amp

Table 1 - Theoretical polarization matrix vs. their measured counterparts.

\begin{tabular}{|l|c|c|}
\hline & Theoretical Polarization Matrix & Measured Polarization Matrix \\
\hline Direct Beam & {$\left[\begin{array}{lll}1 & 0 & 0 \\
0 & 1 & 0 \\
0 & 0 & 1\end{array}\right]$} & {$\left[\begin{array}{lll}0.957 & 0.022 & 0.047 \\
0.041 & 0.959 & 0.083 \\
0.040 & 0.097 & 0.964\end{array}\right]$} \\
\hline Test Solenoid & {$\left[\begin{array}{lll}0.535 & 0.256 & 0.805 \\
0.256 & 0.860 & 0.443 \\
0.805 & 0.443 & 0.395\end{array}\right]$} & {$\left[\begin{array}{lll}0.532 \pm 0.003 & 0.217 \pm 0.004 & 0.878 \pm 0.002 \\
0.299 \pm 0.004 & 0.841 \pm 0.002 & 0.406 \pm 0.004 \\
0.818 \pm 0.003 & 0.472 \pm 0.004 & 0.327 \pm 0.004\end{array}\right]$} \\
\hline
\end{tabular}

\subsection{Conclusion and Future Work}

The comparison of the measured polarization matrix with the theoretical polarization matrix shows that the device is working but has some error associated with it. Some of the components are off and asymmetric within the matrix. This can be explained by some inaccuracies with the angle offset from the solenoid placement, as well as with the assembly of the solenoid itself. The solenoid is encased in mu-metal in an effort to provide solely a vertical field but imperfections in the solenoid can cause field to leak and affect the polarization measurements.

The SNP device has shown some reasonable functionality for the initial testing. Some of the key notes to continue improving the design were on improving the cooling to the precession region, vibration dampening on the support structures, and improving the magnetic shielding. Further testing of the device using the orange cryostat and samples with more interesting magnetic structures will be performed at the hybrid spectrometer (HYSPEC) at the spallation neutron source (SNS) at Oak Ridge National Laboratory (ORNL). For these next tests HYSPEC will be treated as a triple-axis. Lastly, the design for using the SNP device on HYSPEC will continue and some parts for the initial protype have already been fabricated. 


\section{References}

[1] M. Blume, Phys Rev 130 (5), 1670 (1963).

[2] E. Lelievre-Berna, P. J. Brown, F. Tasset, K. Kakurai, M. Takeda, and L. P. Regnault, Physica B 397 (1-2), 120 (2007).W.A. Mozart, Don Giovanni, in proceedings of Mock conference POS (MC2000) 002.

[3] O. Scharpf and H. Capellmann, Phys Status Solidi A 135 (2), 359 (1993).

[4] F. Tasset, Physica B 156, 627 (1989).

[5] T. Wang, S. R. Parnell, W. A. Hamilton, F. Li, A. L. Washington, D. V. Baxter, and R. Pynn, Rev Sci Instrum 87 (3) (2016).

[6] T. Wang, F. Li, S. R. Parnell, W. A. Hamilton, H. Kaiser, A. L. Washington, D. V. Baxter, and R. Pynn, J Phys Conf Ser 528 (2014).

[7] S. R. Parnell, H. Kaiser, A. L. Washington, F. Li, T. Wang, D. V. Baxter, and R. Pynn, Physes Proc 42, 125 (2013).

[8] F. Li, S. R. Parnell, T. Wang, D. V. Baxter, and R. Pynn, International Conference on Polarised Neutrons for Condensed Matter Investigations (Pncmi 2014) 711 (2016).

[9] T. Wang, C. Y. Jiang, H. Z. Bilheux, J. C. Bilheux, L. Crow, L. Robertson, R. J. Taylor, R. Pynn, S. Parnell, W. A. Hamilton, and X. Tong, Appl Phys Lett (in preperation). 